\title{
Research Paper: Occupational Performance Priorities of Children With Cerebral Palsy From the Parents' Perspective
}

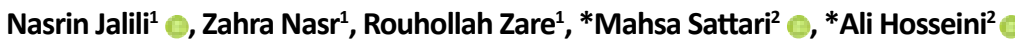

1. Department of Occupational Therapy, School of Rehabilitation, Isfahan University of Medical Sciences, Isfahan, Iran. 2. Department of Occupational Therapy, University of Social Welfare and Rehabilitation Sciences, Tehran, Iran.

\begin{tabular}{|c|c|}
\hline $\begin{array}{l}\text { Use your device to scan } \\
\text { and read the article online }\end{array}$ & \\
\hline 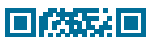 & dtation Jalili N, Nasr Z, Zare R, Sattari M, Hosseini A. [Occupational Performance Priorities of Children With Cerebral Palsy \\
\hline ryts: & From the Parents' Perspective (Persian)]. Archives of Rehabilitation. 2019; 19(4):370-379. \\
\hline 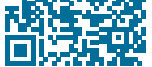 & d - $/$ http://dx.doi.org/10.32598/rj.19.4.370 \\
\hline
\end{tabular}

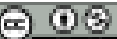

Received: 10 Jun 2018

Accepted: 25 Nov 2018

Available Online: 01 Jan 2019

Keywords:

Canadian occupational performance

measurement, Participation, Clientcentered, Parents of children with Cerebral Palsy

\section{ABSTRACT}

Objective Cerebral Palsy (CP) is a group of non-progressive disorders in a developing brain that results in motor problems and other limitations in daily living activities. The presence of motor functions in these children reduces their participation in daily activities. This is one of the important aspects of the child's health and development, especially the child's physical and psychological development. Participation in social activities promotes life satisfaction. Besides, family has a prominent role in identifying and supplying the child's needs. In other words, family plays an essential role in ensuring the health and well-being of their children. Thus, provisions of growth-related health services has now changed from a child-cantered model into a family-cantered model. As a result, it is very important to understand the parents' priorities about participation of their children in the daily activities and providing appropriate client-center services.

Materials \& Methods In this descriptive-analytic study, 78 children and teenagers with CP aged 3-18 years without psychiatric problems and their parents from Yazd City, Iran had participated. The samples were selected by convenience sampling method, from clients of private and public rehabilitation centers in Yazd Province. In this regard, one state and two private centers were randomly selected and the study samples were selected from the clients referred to the centers. Parents' priorities for participation of their children in activities of daily living were found through semi-structured interviews. In these interviews, we used Canadian occupational performance measure to evaluate the child's level of occupational performance and parents' satisfaction in the three areas of self-care, productiveness, leisure from the parents' perspective. In addition, the level of gross motor performance was evaluated based on the Gross Motor Function Classification System (GMFCS) at five levels. In the Iranian society, the validity of Canadian occupational performance measure for each of three areas of occupational performance has been reported to be between 9.75 and 3.75 and the reliability level for the three domains reported above 0.78 . The statistical analysis of data was performed in SPSS V. 16. Descriptive statistics and odds ratio were used for analyzing the data.

Results the children' Mean \pm SD age was $8.12 \pm 4.22$ years. The first priority reported by the parents was self-care. Mobility was known as the second important priority in everyday life for children. The results obtained from the statistical analysis showed no significant relationship between age and different motor levels $(P \geq 0.05)$

Conclusion Parents reported functional priorities in similar performance aspects by all age groups and GMFCS levels in Cerebral Palsy children. The first priority was related to self-care. This reflects the desire of the parents to children' doing self-care activities, which not only reduces care pressure, but also leads to promotion in social participation and independence in individual life. The next priority was mobility and the reason can be attributed to the fact that children with CP need mobility to manage their daily life activities.

\section{* Corresponding Author:}

\section{Mahsa Satari, PhD. Candidate}

Address: Department of Occupational Therapy, School of Rehabilitation, Isfahan University of Medical Sciences, Isfahan, Iran.

Tel: +98 (912) 6810129

E-Mail: satari_mahsa@yahoo.com

\section{Ali Hosseini, PhD}

Address: Department of Occupational Therapy, University of Social Welfare and Rehabilitation Sciences, Tehran, Iran.

Tel: +98 (912) 6810129

E-Mail: alihosse@gmail.com 


\title{
بررسى اولويتهاى عملكرد كارى در كودكان مبتلا به فلج مغزى: ديدكاه والدين
}

\author{
نسرين جليلى' • زهرا نصر'، روحالله زارع'، • مهسا ستارى' ه • سيد على حسينى'

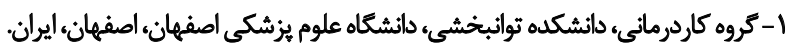

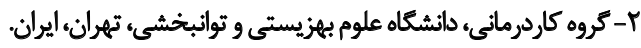

\begin{abstract}
حكS

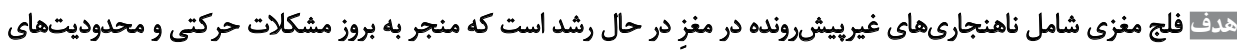

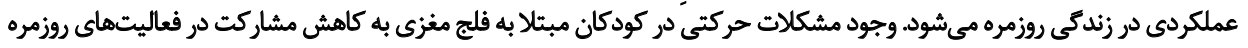

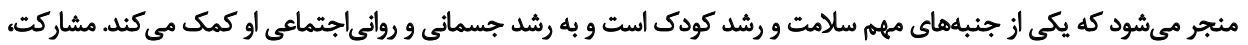

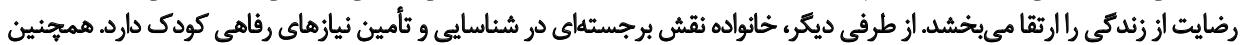

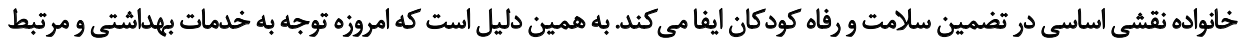

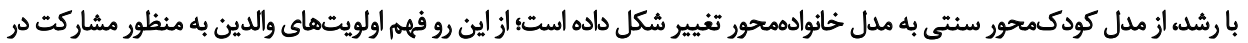

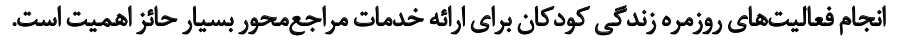

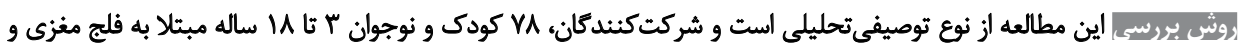

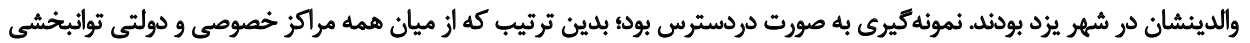

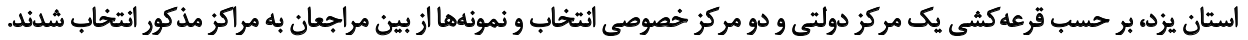

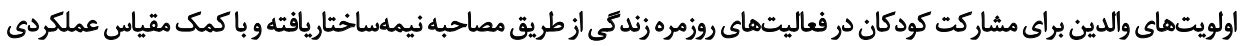

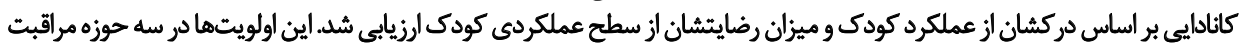

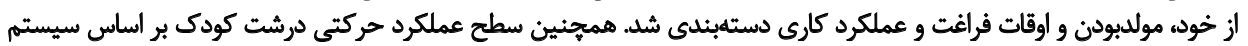

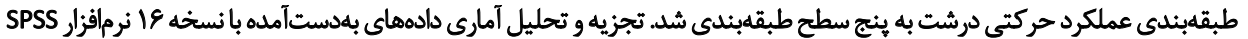

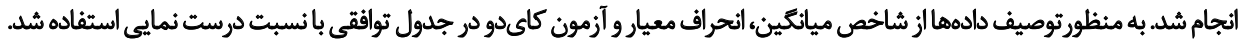

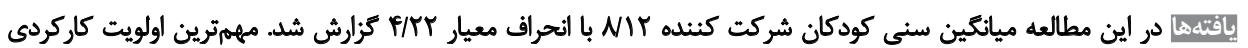

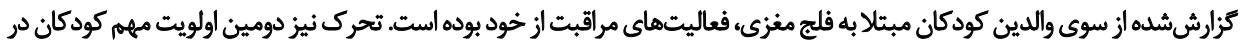

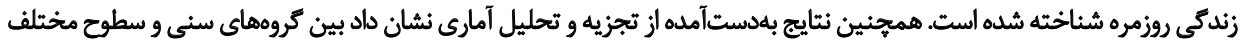

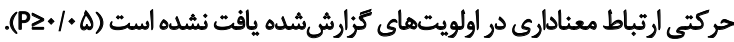

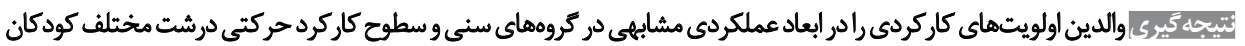

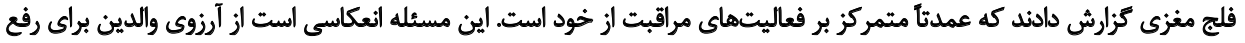

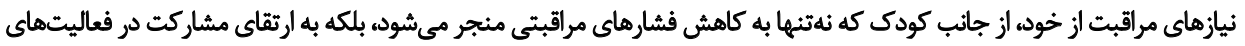

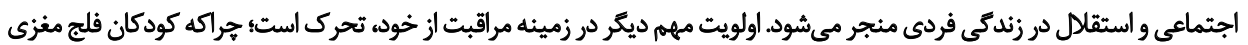

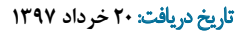

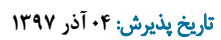

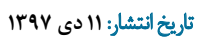

كليدواروها: مدل كاري، مانادايى عملكرد

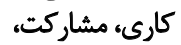
مراجعمحور، والدين مثارين كودكان فلج مغزى مالدين
دكتر سيد على حسينى

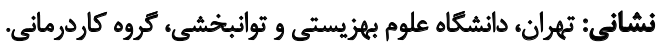
تلفن: alihosse@gmail.com
مهسا ستارى : مئده

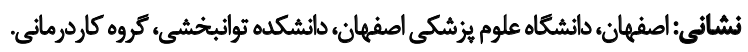
تلفئ راياثامه: satari_mahsa@yahoo.com 
مدل خانوادهمحور تغيير شكل داده است [ • 1]] اين ديدكاه مراجعمدار بر نوعى همكارى اشتراكى بين بيمار

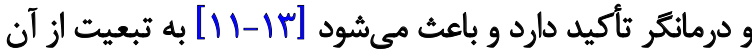

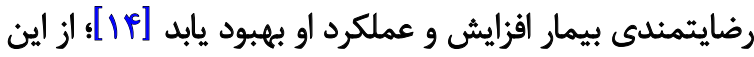

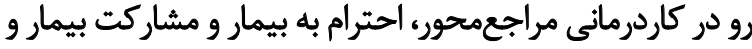

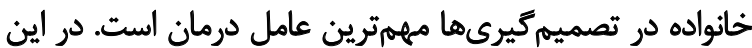

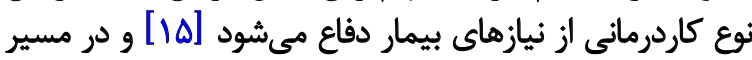

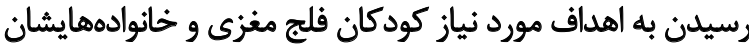
در جريان سيستم مراقبت از سلامت كام برداشته مي كئودان كودكان فلج مغزى در مقايسه با كودكان طبيعى همسال

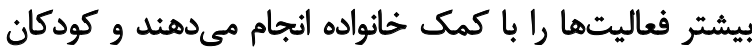

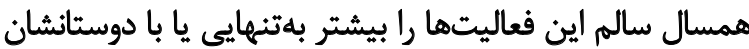

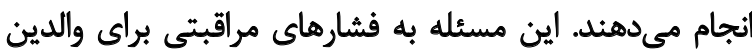

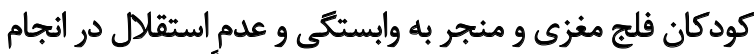

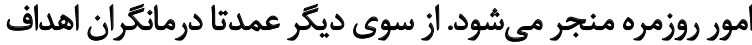

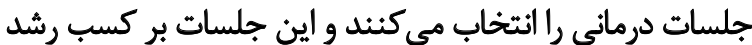
حركتى متمركز هستئد.

با توجه به اينكه والدين بهتر از هر كسى كودى خود را

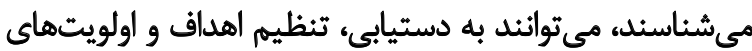

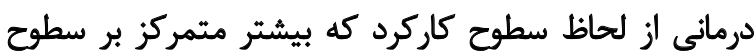

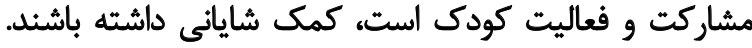

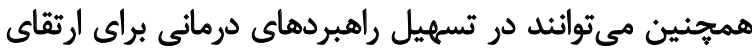

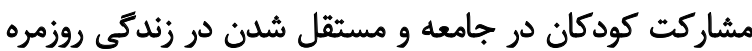

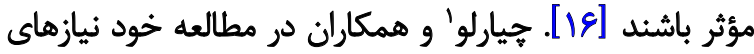

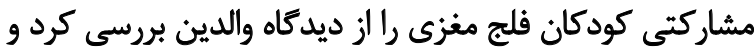

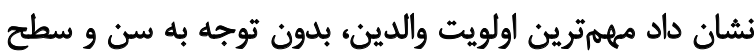

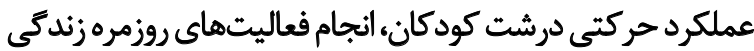
در مسير كسب استقلال است كودكان، [انجام همجينين تأكيد بسيار سازمان جهانى بهداشت بر اهميت

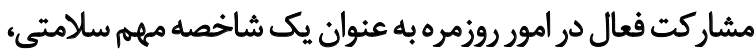
سيستمهاى بهداشتى و اجتماعى جوامع مختلف رئل را بر آن داشتئه

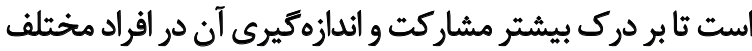

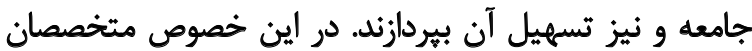

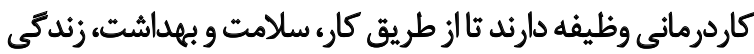

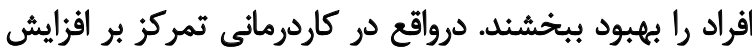

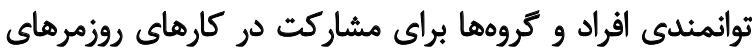

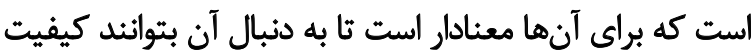
زندگى افراد راتحت تأثير قرار دهند. در ايران مطالعهاى يافت نشد كه اولويت مشاركت كودكان

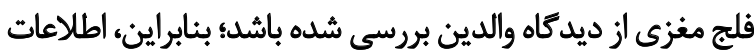

1. Chiarello مelat

فلج مغزى به اختلالات غيرييشرونده در حركت و وضعيت

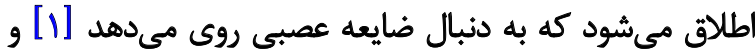

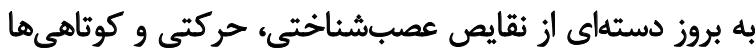

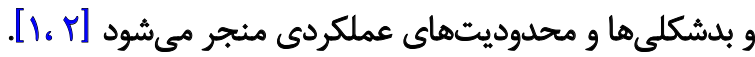

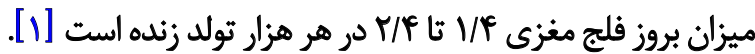

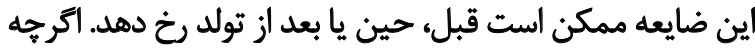

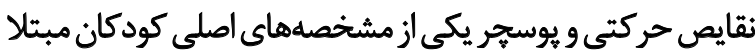

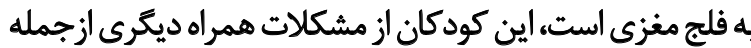

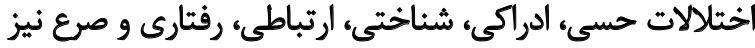

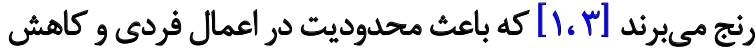

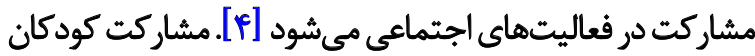

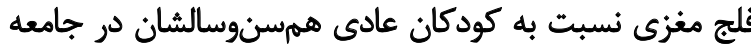

كمتر است [ب] فيت

مطالعات انجامشده در ايران نيز حاكى از آن است كه مشاركت

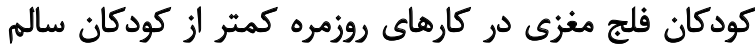

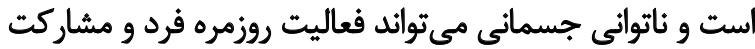

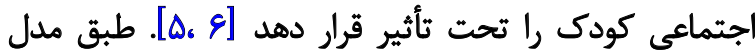

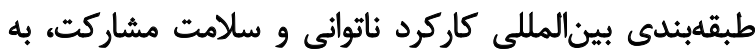

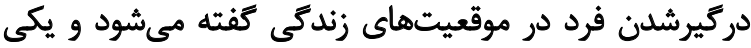

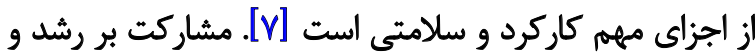

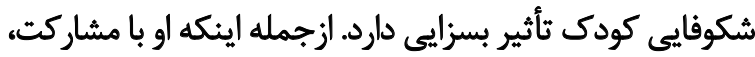

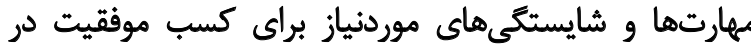

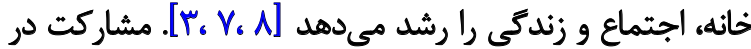

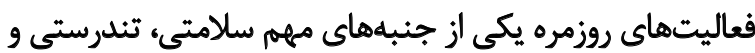

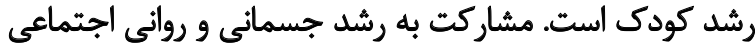

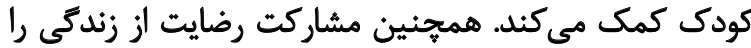
ارتقا مىبخشد [V،

مطالعات نشان مى دهند كودكان ناتوان در مقايسه با كودكان

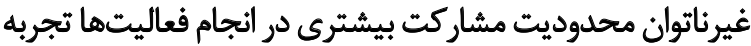

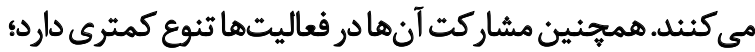

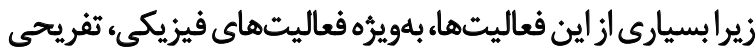

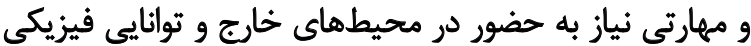

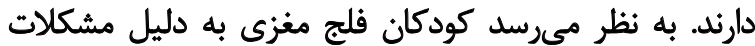

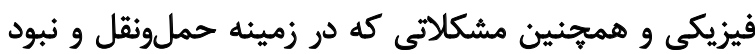

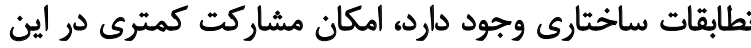

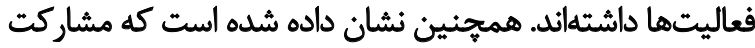

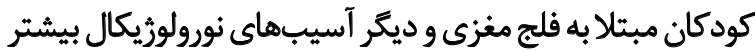

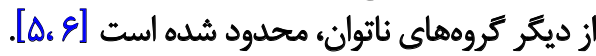
از سوى ديكر خانواده نقش اساسى در تضمين سلامت و رفاه

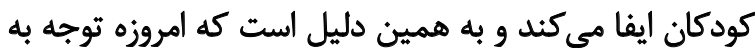

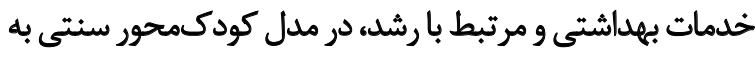


عملكرد حركتى درشت سنجيده شد.

إبؤار هاي الرزيابى

سيستم طبقهبندى عملكرد حركتى درشت، سيستم

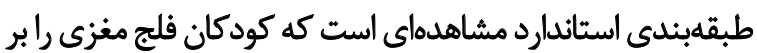

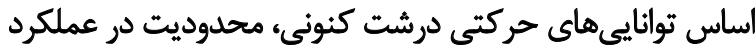

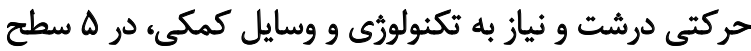

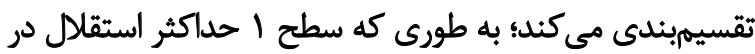

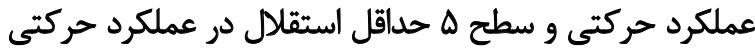

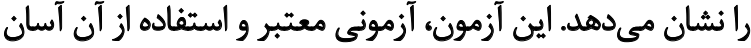

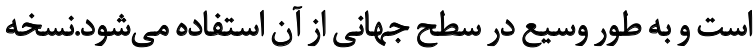

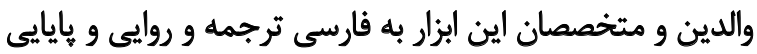

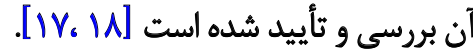
مقياس عملكردى كانادايى مقياس اندازهيرى مراجعمدحور است

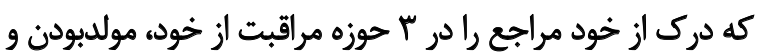

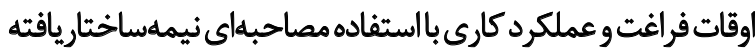

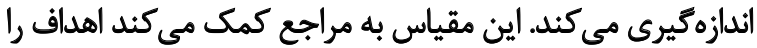

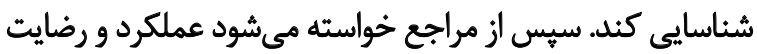

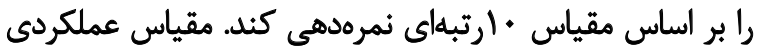

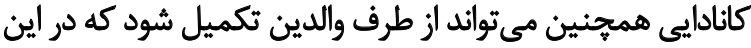
يروهش يرسشنامه راوالدين ير كردهاند [19].

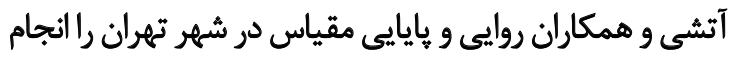

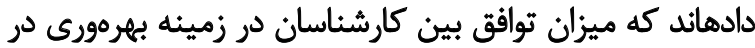

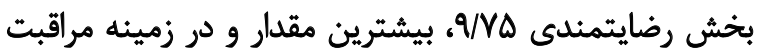

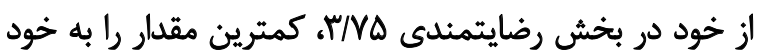

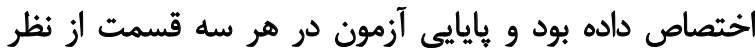

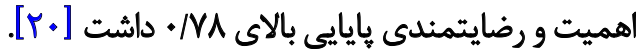

$$
\text { تجزئيه و ثحليل أمارى }
$$

براى تجزيه و تحليل آمارى دادهها از نسخه

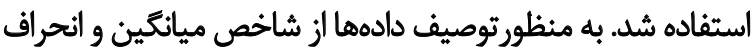

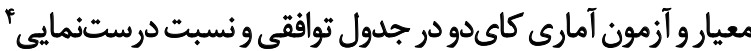

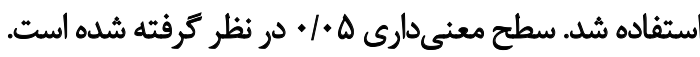

يافتهها

از بين VA نفر كودك، كوجكترين شركت كنينده "اساله و

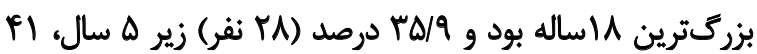

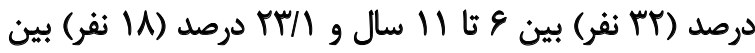

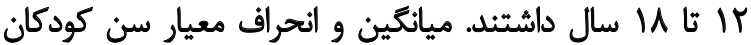

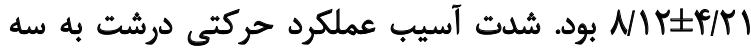

3. Gross Motor Function Classification System (GMFCS) 4. Likelihood Ratio Test
اندكى در اين زميئه در ايران وجود دارد و اولويثهاي درمانى در

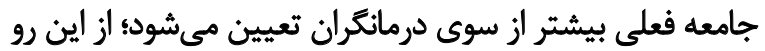

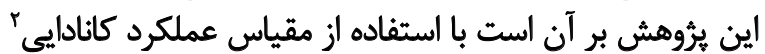

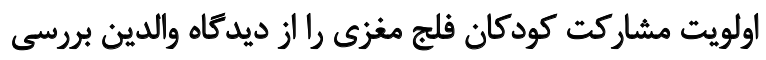

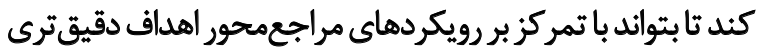

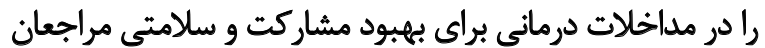
مدنظر قرار دهد. ووش بروسى اين مطالعه از نوع توصيفىتحليلى است كه با هدف بررسى

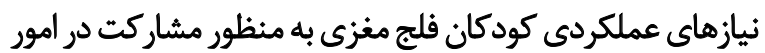

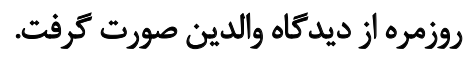

مشار كت كن:نان

جامعه آمارى اين مطالعه، تمامى مادران كودكان مبتلا به فلج

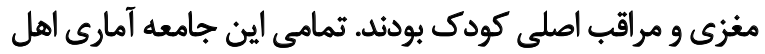

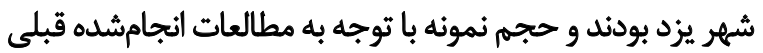
و بر اساس فرمول حجم نمونه تخمين زده شيد.

$$
n=\frac{z_{\left(1 \frac{\alpha}{2}\right)}^{2} P(1-P)}{d^{2}}
$$

روش نمونهكيرى به صورت دردسترس بود؛ بدين ترتيب كه از

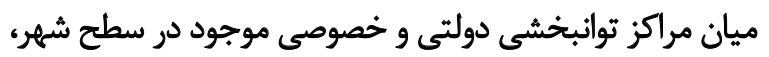

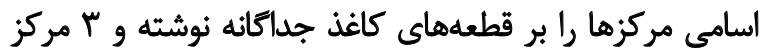

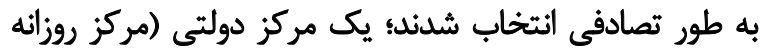
توانيابان) و Y مركز خصوصى (مركز توانبخشى سين سينا و مركز

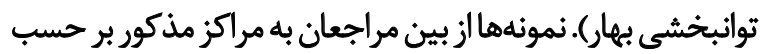

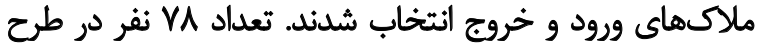
شركت كردند. اين بثروهش در اسفند هوب إ انجام شده است.

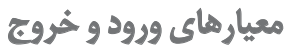

ملاكهاى ورود شامل تشخيص فلج مغزى از سوى يزشك مأبك متخصص مغز و اعصاب كودكان بدون همراهي ماهي مشكليات

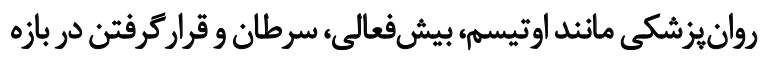

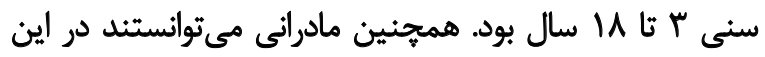

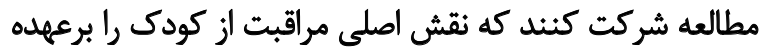

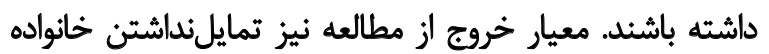

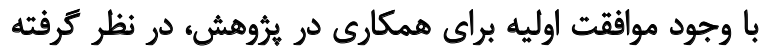

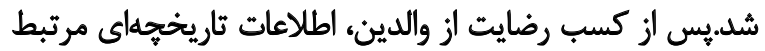

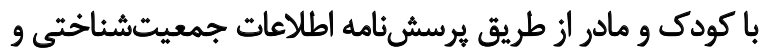

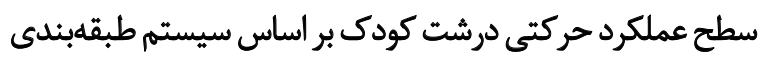

2. Canadian Occupational Performance Measure (COPM) 
جدول ا. توزيع فراوانى اولين و دومين اولويت والدين در ابعاد كلى يرسشنامه COPM به تفكيك كروهاي سنى

\begin{tabular}{|c|c|c|c|c|c|c|}
\hline$P$ & هيجكدام & اوقات فراغت & بهرهورى & مراقبت از خُود & مثغير & اولويت والدين \\
\hline \multirow{3}{*}{. } & - & 1 & $r$ & To & زير هال سال & \multirow{3}{*}{ اولويت اول } \\
\hline & - & $r$ & . & $r$ & צتا 11 سال & \\
\hline & - & 1 & $r$ & 10 & كו تا كاسال & \\
\hline \multirow{3}{*}{. $/ \Delta P$. } & . & $r$ & $r$ & $r$ & زير ه سال & \multirow{3}{*}{ اولويت دوم } \\
\hline & 1 & r & $r$ & $r q$ & وتا 11 سال & \\
\hline & 1 & 1 & $r$ & ir & צו تا هاسال & \\
\hline
\end{tabular}

توانبخننى

جدول r. توزيع فراواني اولين و دومين اولويت والدين در خردهمقياسهاي COPM به تفكيك كروههاي سنى

\begin{tabular}{|c|c|c|c|c|c|c|c|c|c|}
\hline $\mathbf{P}$ & شدن أتمى & تفريحات & تفريحات & مدرسه & مديريتى & عملكردى & شخرقبت & متغير & والدين \\
\hline \multirow{3}{*}{$.1 .0 \mathrm{~A}$} & 1 & - & * & $r$ & - & 11 & 18 & زير ه سال & \multirow{3}{*}{ أولويت اول } \\
\hline & • & 1 & 1 & • & - & 1 & r & عأ الال سال & \\
\hline & + & 1 & • & $r$ & $r$ & $r$ & 11 & ז' & \\
\hline \multirow{3}{*}{. NEY } & - & - & - & $r$ & $r$ & $\Delta$ & 18 & زير ه سال & \multirow{3}{*}{ اولويت دوم } \\
\hline & 1 & $r$ & - & 1 & $r$ & 1 & $M$ & عتا ال سال & \\
\hline & 1 & • & 1 & 1 & $r$ & $r$ & 1 & rائا Aا سال & \\
\hline
\end{tabular}

توانبخننى

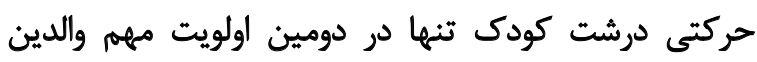

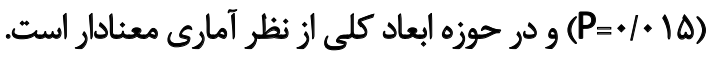

\section{بحث}

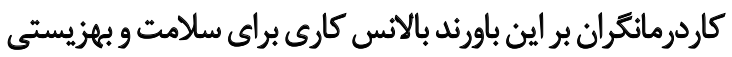

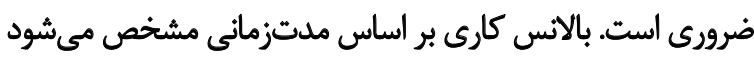

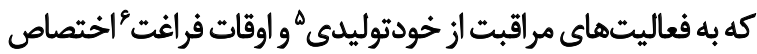

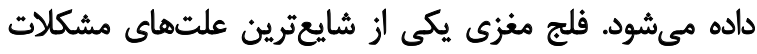

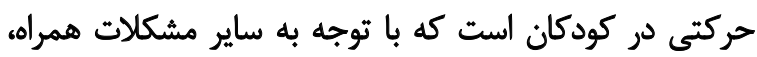

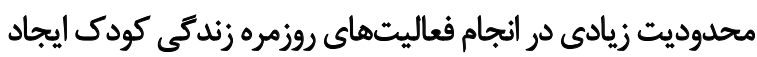

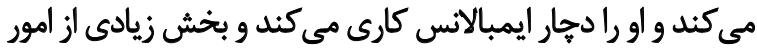

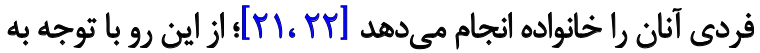

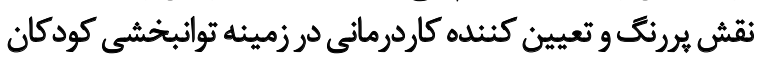

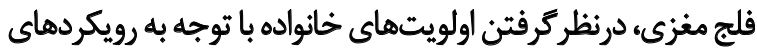

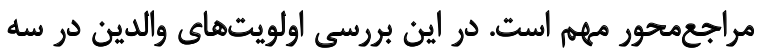

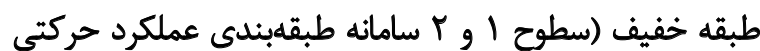

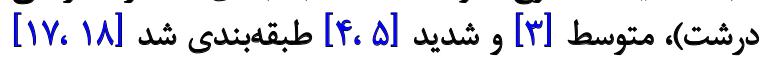

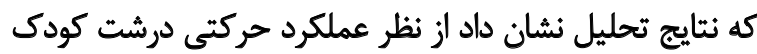

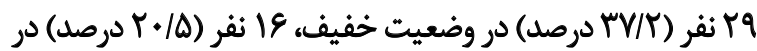

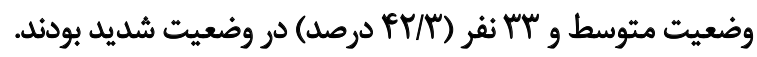
براى بررسى تأثير سن كودى بر اولويت والدين، ابتدا سن

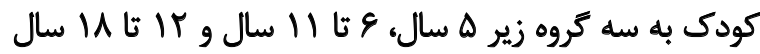

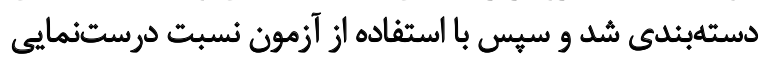

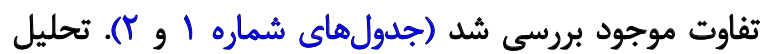

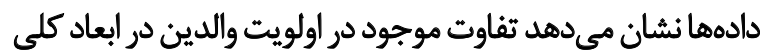

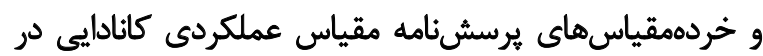

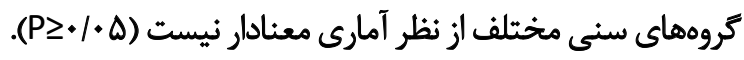

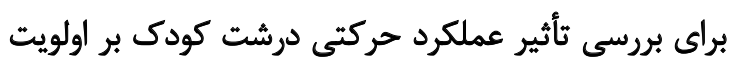

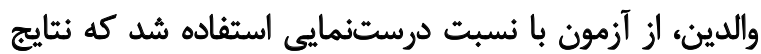

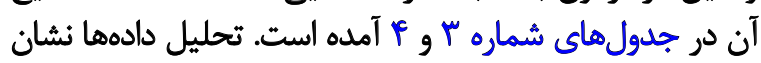
مىدهد تفاوت موجود در اولويت والدين بر حسب شدات تحلي عملكرد 
جدول ץ. توزيع فراوائى اولين و دومين اولويت والدين در ابعاد كلى يرسشنامه COPM به تفكيك عملكرد حركتى درشت كودى

\begin{tabular}{|c|c|c|c|c|c|c|}
\hline$P$ & هيجيجدام & اوقات فراغت & بهرهورى & مراقبث از خود & متغير & اولويث والدين \\
\hline \multirow{3}{*}{. $/ \& \Delta \Delta$} & - & 1 & r & re & خفيف & \multirow{3}{*}{ الولويث اول } \\
\hline & - & $r$ & 1 & ir & مثتوسط & \\
\hline & - & 1 & 1 & $m$ & شديد & \\
\hline \multirow{3}{*}{$+1+10$} & 1 & $r$ & $\wedge$ & in & هُفيف & \multirow{3}{*}{ اولويت دوم } \\
\hline & 1 & • & 1 & if & متوسط & \\
\hline & . & $\Delta$ & 1 & TV & شديد & \\
\hline
\end{tabular}

جدول P. توزيع فراوائى اولين و دومين اولويت والدين در خردهمقياسهاي COPM به تفكيك عملكرد حركتى درشت كودى

\begin{tabular}{|c|c|c|c|c|c|c|c|c|c|}
\hline$P$ & شُتَمن & تفريبحات & تفريحات & مدرسه & اجتماعي & عملكردى & شرخصى & متفير & أولويت والدير \\
\hline \multirow{3}{*}{$=4+4$} & - & 1 & - & $r$ & $r$ & $\wedge$ & 18 & خفيف & \multirow{3}{*}{ الولويت اول } \\
\hline & 1 & 1 & • & 1 & • & $r$ & 1. & هثوسط & \\
\hline & • & • & 1 & 1 & - & 1. & rI & شديد & \\
\hline \multirow{3}{*}{ 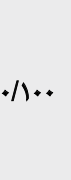 } & 1 & - & 1 & $r$ & $v$ & $r$ & 10 & هُفيفِ & \multirow{3}{*}{ اولويث دوم } \\
\hline & 1 & • & . & • & 1 & $r$ & 1. & متوسطط & \\
\hline & • & $r$ & • & $r$ & 1 & $f$ & זr & شديد & \\
\hline
\end{tabular}

توانبخننى

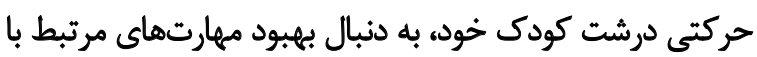

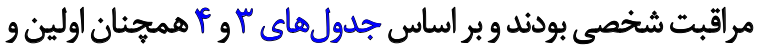

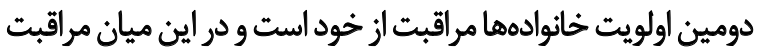

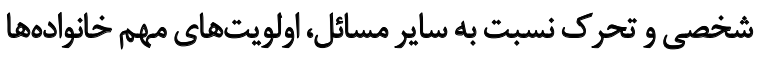

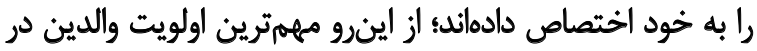

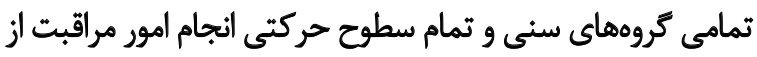

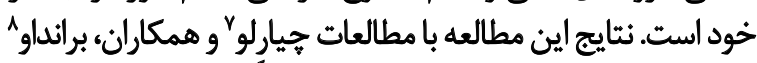

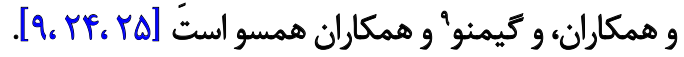

همانطور كه نتايج نشان داد اولويت والدين در ابعاد كلى مشاركت (مراقبت از خود، بهرهورى و اوقات فراغت) براي كود كان

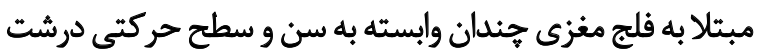

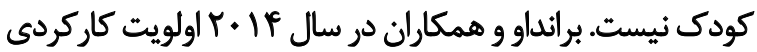

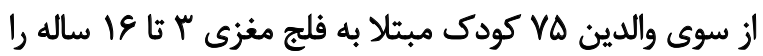

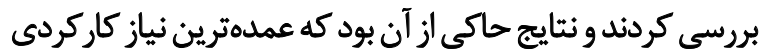

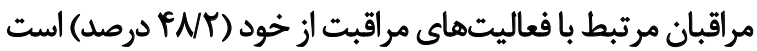

7. Chiarello

8. Brandão

9. Gimeno
زمينه فعاليتهاى روزانه زندكى، فعاليتهاى توليدى و اوقات

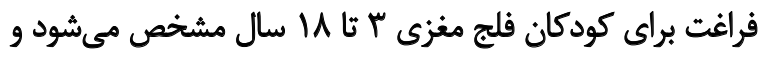

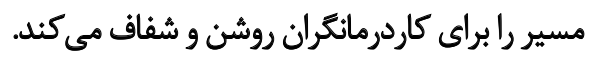
نتايج اين يُروهش نشان داد مراقبت از خود اولين اولويت بيشتر

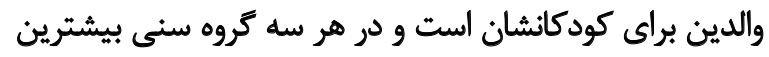

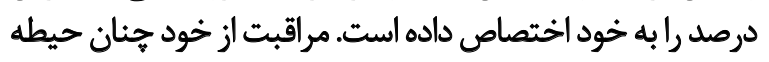

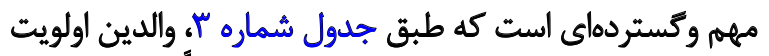

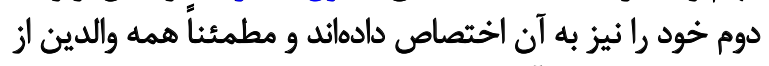
اهميت اين مسئله آكاه هستئد. حيطه مراقبت از خود در اين يزوهش طبث مقياس عملكردى

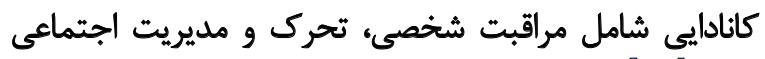

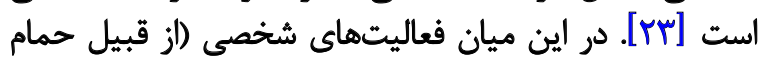

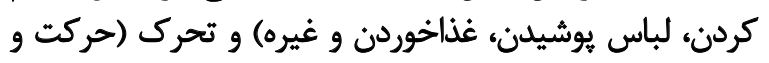

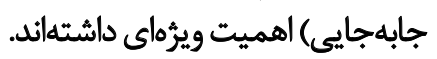

كودكان شركت كننده در اين بررسى بر اساس مقياس طبقهبندى عملكرد حركتى درشت به سه دسته (خفيف، متوسط، شديد)

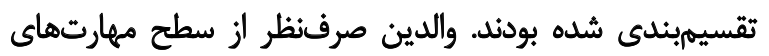


تحرك دومين اولويت والدين در حيطه مراقبت از خود است.

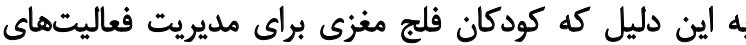

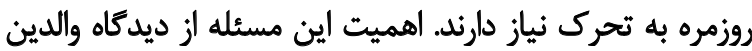

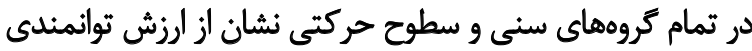

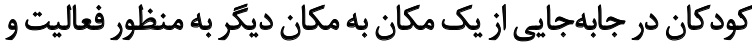

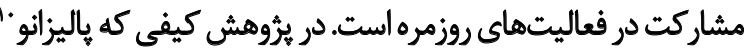

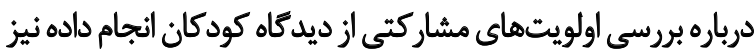

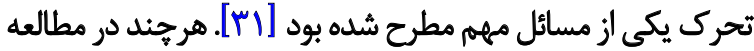

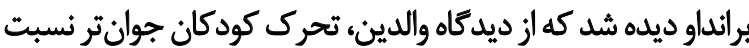

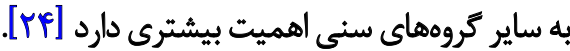

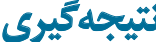

نتّايج نشان داد والدين اولويتهاي كاركردى رادر ابعاد عملكردى

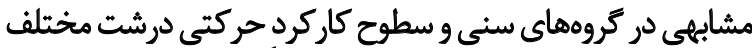

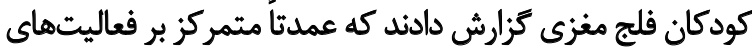

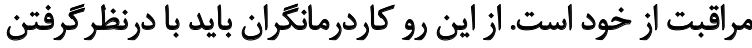

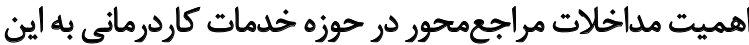

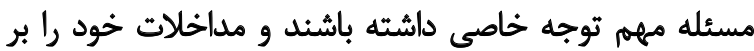

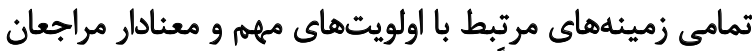

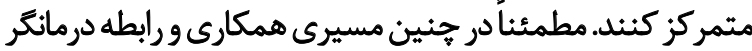

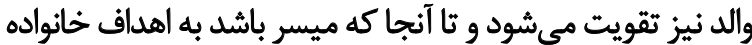

$$
\text { وكودى دست خواهيم يافت. }
$$

از جمله مهمترين محدوديتهايى كه وجود داشت مىتوان

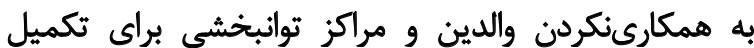

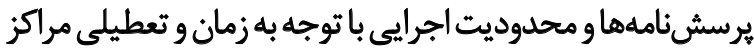

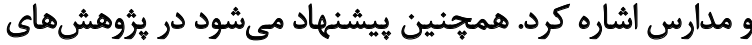
بعدى اولويتهاى مشاركتى از ديد ماه كودى ودي و والد با هم مقايسه

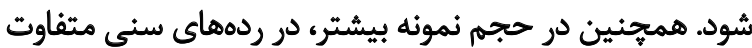

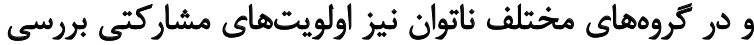

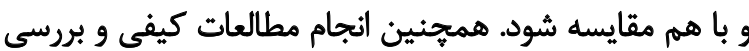

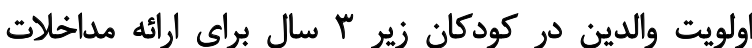

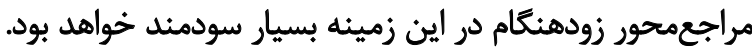

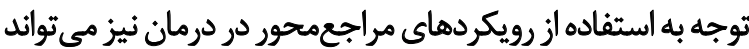

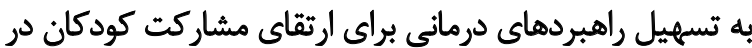

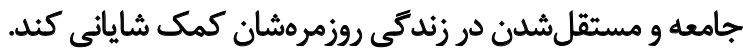

مالاحظات اخلاقي

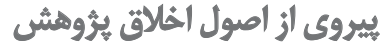

در يروهش حاضر به شركتكنندكان اطمينان داده شد اند

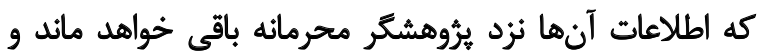

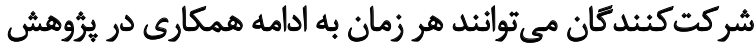

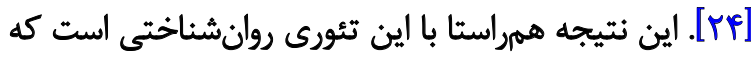

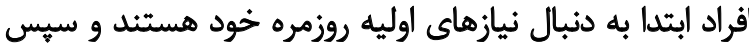

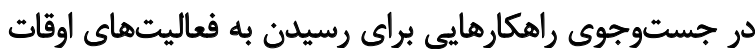

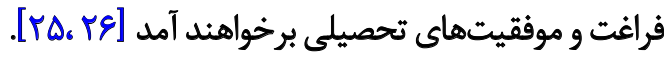
والدين كودكان مبتلا به فلج مغزى، بدون توجه به سن كودى

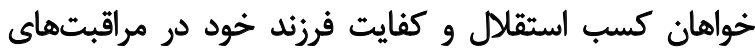

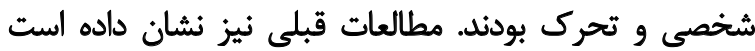

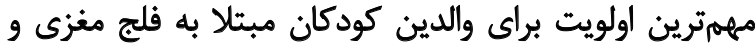

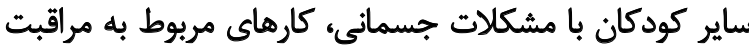

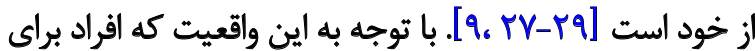

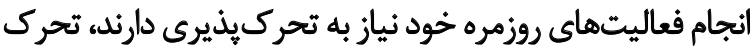

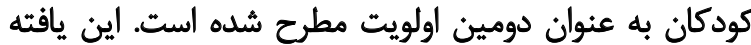

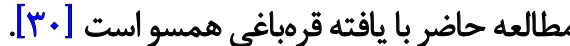
به دليل نقش معنادار مراقبتهاى فردى در استقلال دوران

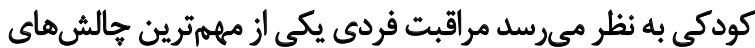

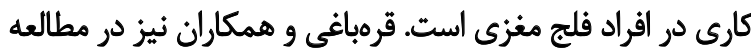

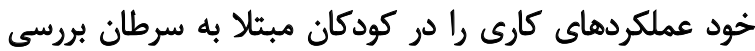

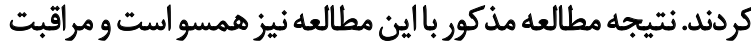

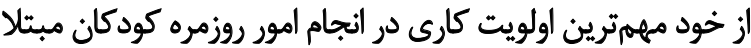

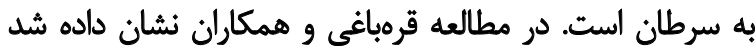

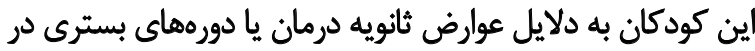

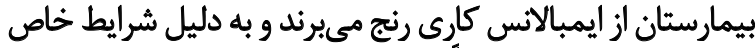

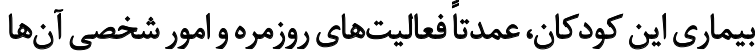

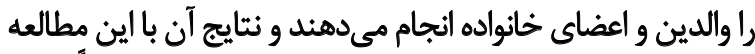

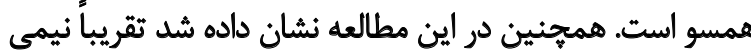

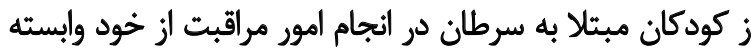

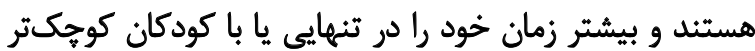

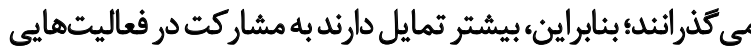

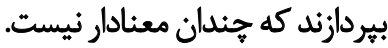

مشاركت محدود يا شركت در فعاليتهايى كه معنادار نيست

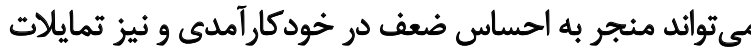

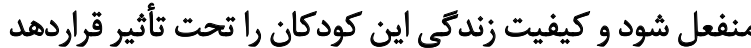

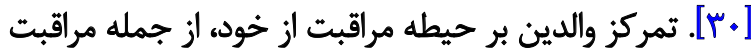

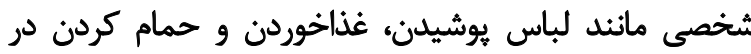

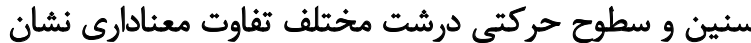

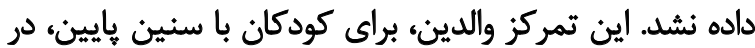

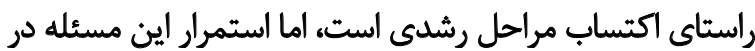

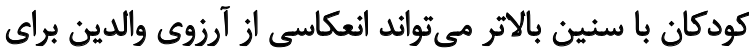

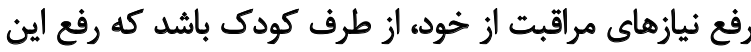

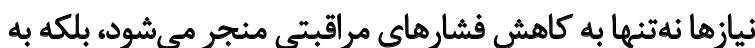

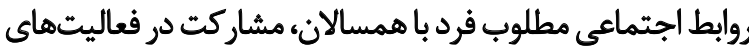
جتماعى و انتقال به يك زندگى مستقل منجر مى همود. 


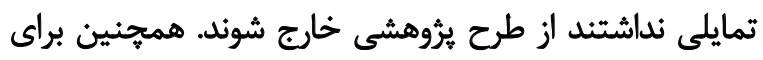

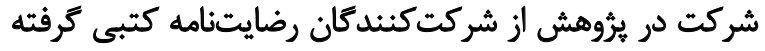

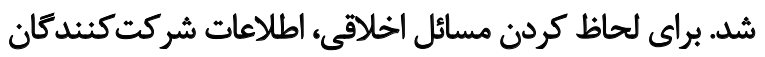

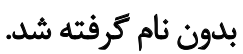

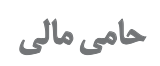

مقاله حاضر حاصل طرح يثوهشى مصوب نيست و در قالب سمينار دانشجويى انجام شده است.

$$
\text { مشاركت نويسند مَّان }
$$

مفهومسازى: نسرين جليلى، سيد على حسينى، مهسا ستارى؛

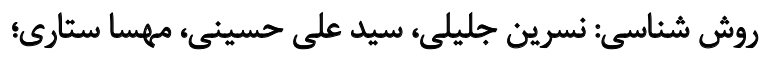

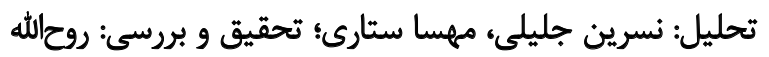

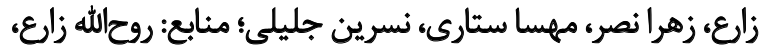

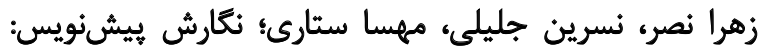

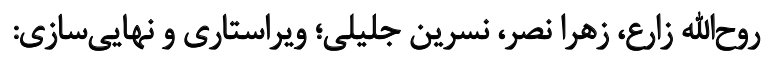

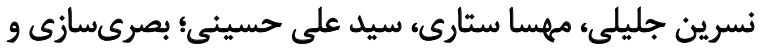

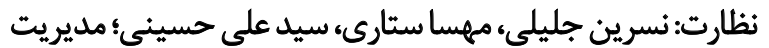

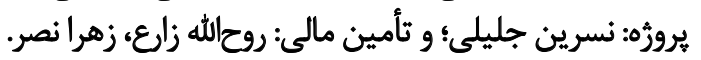

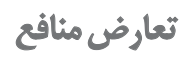

بنابر اظهار نويسندكان، اين مقاله تعارض منافع ندارد. 


\section{References}

[1] Case-Smith J, O'Brien JC. Occupational therapy for children. Amsterdam: Elsevier Health Sciences; 2013.

[2] Rosenbaum P, Paneth N, Leviton A, Goldstein M, Bax M, Damiano $D$, et al. A report: The definition and classification of Cerebral Palsy April 2006. Developmental Medicine and Child Neurology Supplement. 2007; 109(suppl.109):8-14. [PMID]

[3] Nobakht Z, Rassafiani M, Rezasoltani P. [Influence of child's disability on encountering environmental barriers to participation of children with Cerebral Palsy (Persian)]. Journal of Rehabilitation Sciences and Research. 2013; 9(2):286-94.

[4] Jalili N, Godarzi M, Rassafiani M, Haghgoo H, Dalvand H, Farzi $M$. [The influenced factors on quality of life of mothers of children with severe Cerebral Palsy: A survey study (Persian)]. Modern Rehabilitation. 2013; 7(3):40-7.

[5] Rostam Zadeh O, Amini M, Hasani Mehraban A. [Research paper: Comparison of participation of children with Cerebral Palsy aged 4 to 6 in occupations with normal peers (Persian)]. Journal of Rehabilitation Medicine. 2016; 17(3):192-9. [DOI:10.21859/jrehab-1703192]

[6] Hassani Mehrabanm A, Hasani M, Amini M. The comparison of participation in school-aged Cerebral Palsy children and normal peers: A preliminary study. Iranian Journal of Paediatrics. 2016; 26(3):e5303. [DOI:10.5812/ijp.5303]

[7] World Health Organization. International Classification of Functioning, Disability, and Health: Children \& Youth Version: ICF-CY. Geneva: World Health Organization; 2007.

[8] Michelsen SI, Flachs EM, Uldall P, Eriksen EL, McManus V, Parkes J, et al. Frequency of participation of 8-12-year-old children with Cerebral Palsy: A multi-centre cross-sectional European study. European Journal of Paediatric Neurology. 2009; 13(2):165-77. [DOI:10.1016/j.ejpn.2008.03.005] [PMID]

[9] Chiarello LA, Palisano RJ, Maggs JM, Orlin MN, Almasri N, Kang $\mathrm{L}$, et al. Family priorities for activity and participation of children and youth with Cerebral Palsy. Physical Therapy. 2010; 90(9):1254-64. [DOI:10.2522/ptj.20090388] [PMID]

[10] Committee on Children With Disabilities. Role of the pediatrician in family-centered early intervention services. Pediatrics. 2001; 107(5):1155-7. [DOI:10.1542/peds.107.5.1155]

[11] Darrah J, Law M, Pollock N. Family-centered functional therapy-a choice for children with motor dysfunction. Infants \& Young Children. 2001; 13(4):79-87. [DOI:10.1097/00001163200113040-00014]

[12] Lammi BM, Law M. The effects of family-centred functional therapy on the occupational performance of children with Cerebral Palsy. Canadian Journal of Occupational Therapy. 2003; 70(5):285-97. [DOI:10.1177/000841740307000505] [PMID]

[13] Mohamadi F, Sourtiji H, Hosseini MS. [A new approach in rehabilitation of children with Cerebral Palsy (Persian)]. Journal of Research in Rehabilitation Sciences. 2012; 7(5):758-65.

[14] Eyssen I, Beelen A, Dedding C, Cardol M, Dekker J. The reproducibility of the Canadian occupational performance measure. Clinical Rehabilitation. 2005; 19(8):888-94. [DOI:10.1191/0269215505cr883oa] [PMID]

[15] Carswell A, McColl MA, Baptiste S, Law M, Polatajko H, Pollock N. The Canadian occupational performance measure: $\mathrm{A}$ research and clinical literature review. Canadian Journal of Occupational Therapy. 2004; 71(4):210-22. [DOI:10.1177/00084 1740407100406] [PMID]

[16] Law M, Darrah J, Pollock N, Rosenbaum P, Russell D, Walter SD, et al. Focus on Function-a randomized controlled trial comparing two rehabilitation interventions for young children with Cerebral Palsy. BMC Pediatrics. 2007; 7:31. [DOI:10.1186/1471-2431-7-31] [PMID] [PMCID]

[17] Dehghan L, Abdolvahab $M$, Bagheri $H$, Dalvand H, Faghih Zade $S$. [Inter rater reliability of Persian version of gross motor function classification system expanded and revised in patients with Cerebral Palsy (Persian)]. Daneshvar Medicine. 2010; 18(91):37-44

[18] Riahi A, Rassafiani M, Binesh M. [The cross-cultural validation and test-retest and inter-rater reliability of the Persian translation of parent version of the gross motor function classification system for children with Cerebral Palsy (Persian)]. Archives of Rehabilitation. 2013; 13(5):25-30.

[19] Law M, Stewart D. Test retest reliability of the COPM with children. Ontario: Occupational Therapy University School of Rehabilitation; 1996

[20] Atashi N, Aboutalebi S, Heidari M, Hosseini SA. Reliability of the Persian version of Canadian occupational performance measure for Iranian elderly population. Iranian Rehabilitation Journal. 2010; 8(12):26-30.

[21] Rassafiani M, Sahaf R. Hypertonicity in children with Cerebra Palsy: A new perspective. Iranian Rehabilitation Journal. 2011; 9:66-74.

[22] Soleimani F, Vameghi R, Rassafiani M, Akbar Fahimi $N$, Nobakht Z. Cerebral Palsy: Motor types, gross motor function and associated disorders. Iranian Rehabilitation Journal. 2011; 9:21-31.

[23] Law M, Baptiste S, Carswell A, McColl MA, Polatajko H, Pollock N. Canadian Occupational Performance Measure (COPM). Ottawa, Ontario: Canadian Association of Occupational Therapists; 1998.

[24] Brandão MB, Oliveira RH, Mancini MC. Functional priorities reported by parents of children with Cerebral Palsy: Contribution to the pediatric rehabilitation process. Brazilian Journal of Physical Therapy. 2014; 18(6):563-71. [DOI:10.1590/bjptrbf.2014.0064] [PMID] [PMCID]

[25] Gimeno H, Gordon A, Tustin K, Lin JP. Functional priorities in daily life for children and young people with dystonic movement disorders and their families. European Journal of Paediatric Neurology. 2013; 17(2):161-8.

[26] Maslow AH. A theory of human motivation. Psychological Review. 1943; 50(4):370-96. [DOI:10.1037/h0054346]

[27] Østensj $\varnothing$ S, Øien I, Fallang B. Goal-oriented rehabilitation of preschoolers with Cerebral Palsy-a multi-case study of combined use of the Canadian Occupational Performance 
Measure (COPM) and the Goal Attainment Scaling (GAS). Developmental Neurorehabilitation. 2008; 11(4):252-9. [DOI:10.1080/17518420802525500] [PMID]

[28] Pollock N, Stewart D. Occupational performance needs of school-aged children with physical disabilities in the community. Physical \& Occupational Therapy in Pediatrics. 1998; 18(1):55-68. [DOI:10.1080/J006v18n01_04]

[29] Verkerk GJ, Wolf MJM, Louwers AM, Meester Delver A, Nollet F. The reproducibility and validity of the Canadian Occupational Performance Measure in parents of children with disabilities. Clinical Rehabilitation. 2006; 20(11):980-8. [DOI:10.1177/0269215506070703] [PMID]

[30] Gharebaghy S, Mirbagheri S, Khazaeli Kh. Occupational performance in children aged 6 to 13 Years with Cancer. Function and Disability Journal. 2018; 1(1):44-50

[31] Palisano RJ, Shimmell LJ, Stewart D, Lawless JJ, Rosenbaum PL, Russell DJ. Mobility experiences of adolescents with Cerebral Palsy. Physical \& Occupational Therapy in Pediatrics. 2009; 29(2):133-53. [DOI:10.1080/01942630902784746] [PMID] 